\title{
Attenuated serum adiponectin levels are associated with disease severity in patients with non-traumatic osteonecrosis of the femoral head
}

This article was published in the following Dove Press journal: Journal of Pain Research

3 October 2017

Number of times this article has been viewed

\author{
Yong-Bin Yue ${ }^{1,2}$ \\ Xin Liu $^{2}$ \\ Biao-Fang Wei ${ }^{2}$ \\ 'Guangzhou University of Chinese \\ Medicine, Guangzhou, Guangdong \\ Province, ${ }^{2}$ Department of \\ Orthopedics, Linyi People's Hospital, \\ Linyi, Shandong Province, People's \\ Republic of China
}

Objective: Decreased adiponectin (APN) levels have been detected in patients with osteonecrosis of the femoral head (ONFH). The scope of this study was aimed to explore the relationship between serum APN levels and disease severity in nontraumatic ONFH patients.

Patients and methods: Ninety two nontraumatic ONFH patients and 92 healthy controls were enrolled for this study following the estimation of sample size. Serum APN concentrations were examined by enzyme-linked immunosorbent assay (ELISA). The radiographic progression was determined by Ficat grading system. The clinical severity was evaluated by visual analog scale (VAS), Harris hip scores (HSSs) and Western Ontario and McMaster University Osteoarthritis Index (WOMAC) scores.

Results: Serum APN levels were significantly lower in ONFH patients than in healthy controls. Serum APN levels were significantly lower in patients with Ficat stage 4 ONFH than in patients with stage $3 \mathrm{ONFH}$, and APN levels in patients with stage 3 were lower compared with stage 2 . Serum APN levels were negatively correlated with the Ficat grading system. In addition, serum APN levels were also negatively related to VAS and WOMAC scores and positively associated with HSSs.

Conclusion: Decreased serum APN levels may reflect disease severity of nontraumatic ONFH. Keywords: adiponectin, radiological severity, symptomatic severity, nontraumatic osteonecrosis of the femoral head

\section{Introduction}

Nontraumatic osteonecrosis of the femoral head (ONFH) is a debilitating disorder with a considerably high incidence in the young and middle-aged individuals and consequently brings huge economic burden around the world. ${ }^{1}$ It is mainly characterized by insufficient supply of blood, death of osteocytes and bone marrow cells as well as progressive femoral head damage. Approximately 10,000-20,000 new patients with ONFH are diagnosed each year in the US, and there are 300,000-600,000 people having the disease. ${ }^{2}$ Although etiological and pathological mechanisms of ONFH have not been yet thoroughly investigated, the estimated frequency of the three most frequent risk factors for ONFH includes alcohol (20\%-40\%), corticosteroid therapy $(35 \%-40 \%)$, and idiopathic $(20 \%-40 \%))^{3}$

Up to the present, nontraumatic ONFH is mostly diagnosed according to the radiographic manifestations as well as complaints of pain and impaired function. ${ }^{4}$ However, radiographic changes including cystic or osteosclerotic alterations, subchondral collapse, and joint space narrowing are almost signs of medium or late stage..$^{5}$ In addition,
Correspondence: Biao-Fang Wei Department of Orthopedics, Linyi People's Hospital, Jie Fang Road East, No.27 Linyi 276003, Shandong, People's Republic of China

Emailwbflyhip@I63.com 
there are currently no definite means to clinically monitor the progression of nontraumatic ONFH or the efficacy of operative or pharmacological treatment. Therefore, there is an urgent need for more effective, reliable and quantitative methods to monitor early nontraumatic ONFH progression and treatment effects.

Biomarkers are validated tools that can predict disease prognosis and assess the therapeutic effects. The application of novel and reliable biochemical markers in recent years has been widely used. With regard to nontraumatic ONFH, analysis of serum biomarkers could serve as a clinically relevant method to monitor ONFH progression before signs of bone necrosis are visible by radiograph. ${ }^{6}$

Adiponectin (APN) is the most abundantly secreted adipokine from adipose tissue that circulates at high concentrations in healthy individuals. ${ }^{7}$ APN can act on various tissues to perform insulin-sensitizing, anti-inflammatory, antioxidative, and antiatherogenic effects. ${ }^{8}$ Recent studies have shown APN as an important modulator in the regulation of bone metabolism. ${ }^{9}$ Oshima et $\mathrm{al}^{10}$ found that APN could inhibit osteoclastogenesis and promote osteoblastogenesis to further increase bone mass. In another study, Shinoda et $\mathrm{al}^{11}$ observed that APN could act on bone formation through different endocrine pathways. One previous study has identified that lower APN levels were detected in nontraumatic ONFH patients compared with healthy controls. ${ }^{12}$ APN functions both peripherally and centrally at the spinal cord level to modulate pain and peripheral inflammation through activation of APN receptors. ${ }^{13}$

All these works mentioned earlier suggest that APN may play a pivotal role in protecting nontraumatic ONFH patients against bone necrosis. However, to our knowledge, there were no studies available exploring the association of circulating APN levels and disease severity in ONFH patients. Therefore, the purpose of the present study was to study the serum APN levels that may be potential protective biomarkers of nontraumatic ONFH.

\section{Patients and methods}

\section{Study subjects}

In all, 92 nontraumatic ONFH patients treated in our hospital from February 2016 to March 2017 were enrolled in the study. Additionally, 92 sex- and age-matched healthy individuals receiving body check during the same period were recruited. The patients were evaluated in terms of age, sex, body mass index (BMI), degree of pain, duration and progression of symptoms, and any history of alcohol and corticosteroid used (Table 1). The exclusion criteria of ONFH patients were history or evidence of metabolic bone diseases, including bone tumors, malignant tumors with bone metastasis, hyperor hypoparathyroidism, Paget's disease, or renal osteodystrophy. The eligibility criteria of the healthy control subjects were: 1) undergoing physical examination in our hospital during the same time; 2) no basic disease was found; and 3) age range from $35-55$ years. The study was approved by the ethics committee of Linyi People's Hospital (LW2017001). All patients were notified and signed the informed consent.

\section{Laboratory examination}

Venous blood samples were obtained early morning after an overnight fast. Serum was separated by centrifugation at $1500 \times \mathrm{g}$ for $15 \mathrm{~min}$ at $4^{\circ} \mathrm{C}$ and frozen at $-80^{\circ} \mathrm{C}$ until analyses. Serum APN concentrations were determined using commercial enzyme-linked immunosorbent assay (ELISA) kits (Santa Cruz Biotechnology, Santa Cruz, CA, USA). The assays had intra-assay coefficients of variation $4.8 \%$ and inter-assay coefficients of variation $5.3 \%$. All the examinations were repeated three times.

\section{Radiographic progression assessment}

The normal anteroposterior weight-bearing X-ray pelvic radiograph was taken for all the patients. The severity of radiographic progression of ONFH was evaluated using the classical Ficat system: ${ }^{14}$ stage 1 - normal X-ray imaging; stage 2 - normal femoral head contour, but with evidence of bone remodeling like cystic or osteosclerotic regions; stage 3 - evidence of subchondral collapse or flattening of the femoral head; and stage 4 - narrowing of the joint space with secondary degenerative changes in the acetabulum, such as cysts, osteophytes, and cartilage destruction. The grade used for analysis was the higher of the two hips. In this study, patients with Ficat grading $\geq 2$ were enrolled. The results of radiographs were read and evaluated by one experienced radiologist in our hospital who was blinded to the selection of subjects.

\section{Definition of symptomatic severity}

The visual analog scale (VAS) was used to assess the degree of pain, ranging from 0 to 10 , where 0 suggested no pain at all and 10 indicated extreme pain. The symptomatic severity of ONFH was determined according to Harris hip score (HSS) and Western Ontario and McMaster University Osteoarthritis Index (WOMAC) score. The HSS is a clinician-based tool to measure the outcomes following total hip arthroplasty, ${ }^{15}$ and it is also commonly used to evaluate the functional ability of hip-related disease including ONFH. ${ }^{16}$ The WOMAC score 
system is a validated instrument designed specifically for the assessment of pain and function in osteoarthritis (OA) of both the knee and hip. ${ }^{17}$ Both of the HSS and WOMAC scores are widely used based on clinical evidence and experience. ${ }^{18,19}$ The HSS and WOMAC scores were recorded by experienced physicians.

\section{Post hoc statistical power calculation}

Statistic power (1- $\beta$ ) was calculated by using Power Analysis and Sample Size (PASS) 2008 Statistical Software (Kaysville, UT, USA) using the formula given in the following according to the obtained data of different mean APN levels, standard error, and enrolled numbers of patients in each group. ${ }^{20}$ Statistic power was regarded strong when $>0.8$. The detailed calculation formula is listed as follows:

$$
\begin{aligned}
& 1-\beta=\phi\left(z-z_{1-\alpha / 2}\right)+\phi\left(-z-z_{1-\alpha / 2}\right), \\
& z=\left(\mu_{\mathrm{A}}-\mu_{\mathrm{B}}\right) / \sigma\left(\frac{1}{n_{A}}+\frac{1}{n_{B}}\right)
\end{aligned}
$$

\section{Statistical analysis}

Statistical analysis was performed with IBM SPSS ver. 20.0 (IBM Corporation, Armonk, NY, USA). All obtained data were expressed as mean \pm SD or median. Normal data distribution was checked by the Kolmogorov-Smirnov test. APN levels for patients with different Ficat grades were compared by means of one-way analysis of variance (ANOVA). Bartlett's test was utilized to examine the homogeneity of group variances, followed with Tukey's or Tamhane's post hoc tests. Correlations were analyzed with the Pearson or Spearman analysis between APN levels and disease severity, where appropriate. On the basis of the knowledge that APN blood levels are affected by age, sex, and BMI, the relationship between serum APN levels and other variables was calculated by partial correlation. The correlation coefficient between 0.25 and 0.49 was regarded as weak, 0.5 and 0.74 as moderate, and 0.75 and 1 as strong. Statistical significance was fixed at $P<0.05$ for all tests performed.

\section{Results}

\section{Characteristics of subjects}

The ONFH group consisted of 42 men and 50 women, aged $24-57$ years ( $46.3 \pm 7.8$ years) with the mean BMI of $23.6 \pm 2.3 \mathrm{~kg} / \mathrm{m}^{2}$. The distribution of Ficat stages was 30 in stage 2, 34 in stage 3 , and 28 in stage 4 . The healthy control group consisted of 40 men and 52 women, aged 21-55 years (44.7 \pm 8.2 years) with the mean BMI of $22.9 \pm 2.7 \mathrm{~kg} / \mathrm{m}^{2}$. No significant differences were observed in sex $(P=0.882)$, mean age $(P=0.316)$, and BMI $(P=0.442 ;$ Table 1$)$. The statistical power was $\sim 1$ after calculation, suggesting that the sample size of 92 was sufficient to obtain the conclusion (Figure 1).

\section{Circulating APN levels in ONFH patients}

Serum APN levels in patients with nontraumatic ONFH were significantly lower than those in the healthy control group $(7.32 \pm 1.06 \mu \mathrm{g} / \mathrm{mL}$ vs. $9.18 \pm 1.33 \mu \mathrm{g} / \mathrm{mL}, P<0.001$; Figure 2A). A total of 43 patients used steroid before, and 34 patients had a history of alcohol consumption. The remaining 15 patients were regarded as idiopathic group. No significant differences were found among the three groups $(7.49 \pm 1.04 \mu \mathrm{g} / \mathrm{mL}, 7.21 \pm 0.99 \mu \mathrm{g} / \mathrm{mL}$, and $7.16 \pm 0.99 \mu \mathrm{g} / \mathrm{mL}$, respectively, $P=0.380$; Figure $2 \mathrm{~B}$ ).

Table I Demographic characteristics between ONFH patients and healthy controls

\begin{tabular}{llll}
\hline Characteristic & $\begin{array}{l}\text { ONFH } \\
\text { patients } \\
(\mathbf{n = 9 2 )}\end{array}$ & $\begin{array}{l}\text { Healthy } \\
\text { controls } \\
(\mathbf{n = 9 2 )}\end{array}$ & P-value \\
\hline Age (years) & $46.3 \pm 7.8$ & $44.7 \pm 8.2$ & 0.316 \\
Sex (F/M) & $42 / 50$ & $40 / 52$ & 0.882 \\
BMI (kg/m $\left.{ }^{2}\right)$ & $23.6 \pm 2.3$ & $22.9 \pm 2.7$ & 0.442 \\
VAS score & $5(2-8)$ & - & \\
HSS & $42(22-69)$ & - & \\
WOMAC score & $60(44-90)$ & - & \\
Ficat grading $(2 / 3 / 4)$ & $30 / 34 / 28$ & - & \\
Steroid/alcohol/idiopathic $(\mathrm{n})$ & $43 / 34 / 15$ & & \\
Serum APN levels $(\mu \mathrm{g} / \mathrm{mL})$ & $7.32 \pm 1.06$ & $9.18 \pm 1.33$ & $<0.001$ \\
\hline
\end{tabular}

Note: Basic values, serum APN levels, VAS score, HSS, and WOMAC score are expressed as mean value $\pm \mathrm{SD}$ or median (interquartile range).

Abbreviations: ONFH, osteonecrosis of the femoral head; F, female; M, male; BMI, body mass index; VAS, visual analog scale; HSS, Harris hip score; WOMAC, Western Ontario and McMaster University Osteoarthritis Index; APN, adiponectin.

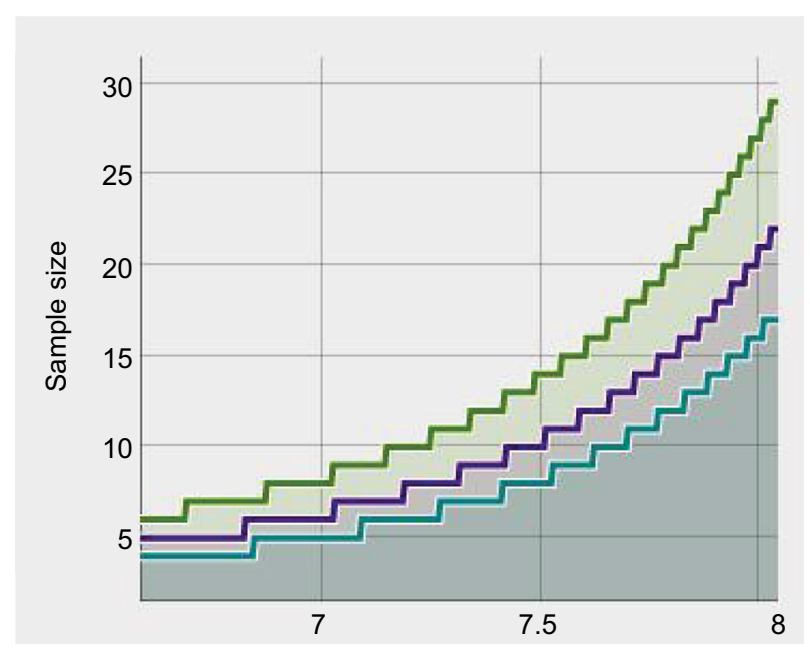

Figure I Statistic power determined by mean and sample size. Statistic power: green line for 0.9 , purple line for 0.8 , and blue line for 0.7 . 

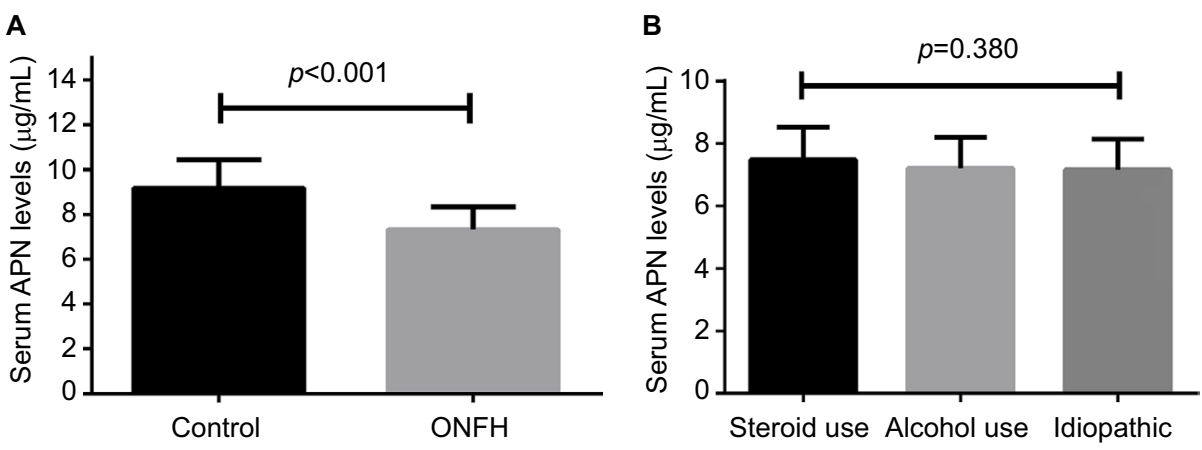

Figure 2 (A) Comparison of serum APN levels between ONFH patients and healthy controls. (B) Comparison of serum APN levels among different steroid-use, alcoholuse, and idiopathic groups.

Abbreviations: APN, adiponectin; ONFH, osteonecrosis of the femoral head.

\section{Association of serum APN levels with Ficat grades}

The circulating APN levels of 92 nontraumatic ONFH patients with different Ficat grades are depicted in Table 2. Accordingly, the ONFH patients were classified into three groups according to the Ficat grading system. The ONFH group included 30 with grade 2, 34 with grade 3, and 28 with grade 4 . ONFH patients with Ficat grade 4 had significantly lower circulating APN levels than those with grades 2 and $3(6.69 \pm 0.12 \mu \mathrm{g} / \mathrm{mL}$ vs. $7.13 \pm 0.13 \mu \mathrm{g} /$ $\mathrm{mL}, P<0.001$ and $6.69 \pm 0.12 \mu \mathrm{g} / \mathrm{mL}$ vs. $8.13 \pm 0.19 \mu \mathrm{g} / \mathrm{mL}$, $P=0.02$, respectively). ONFH patients with grade 3 demonstrated significantly attenuated concentrations of circulating APN compared with those with grade $2(7.13 \pm 0.13 \mu \mathrm{g} / \mathrm{mL}$ vs. $8.13 \pm 0.19 \mu \mathrm{g} / \mathrm{mL}, P<0.001$; Figure $3 \mathrm{~A}$ ). The APN levels in serum were negatively associated with Ficat grading $(r=-0.566, P<0.001)$ by Spearman's correlation analysis (Table 2 and Figures 1 and 3B).

\section{Association of serum APN levels with clinical severity}

We further explored the association of serum APN levels with clinical severity determined by VAS scores, WOMAC scores and HSSs, respectively. As demonstrated in Figure 4, APN levels in serum were negatively correlated with VAS scores $(r=-0.555, P<0.001$; Figure $4 \mathrm{~A})$ and WOMAC scores ( $r=-0.310, P=0.003$; Figure $4 \mathrm{C}$ ) and positively associated with HSSs $(r=0.404, P<0.001$; Figure 4B). All the associations remained significant after adjusting for age and BMI (Table 2).

\section{Discussion}

Our cross-sectional study investigated the relationship between circulating APN levels and disease severity in nontraumatic ONFH patients. Notably, we found that serum
Table 2 Correlations of serum APN levels with anthropometric parameters, Ficat grading, HSSs, VAS scores, WOMAC scores in nontraumatic ONFH patients adjusted by age and BMI

\begin{tabular}{|c|c|c|c|c|}
\hline \multirow[t]{2}{*}{ Variables } & \multicolumn{2}{|c|}{$\begin{array}{l}\text { Serum APN levels } \\
(\mu \mathrm{g} / \mathrm{mL})\end{array}$} & \multicolumn{2}{|c|}{$\begin{array}{l}\text { Serum APN levels } \\
(\mu \mathrm{g} / \mathrm{mL})^{\mathrm{a}}\end{array}$} \\
\hline & $r$ & $P$ & $r$ & $P$ \\
\hline BMI & 0.037 & $>0.05$ & - & \\
\hline Age (years) & 0.102 & $>0.05$ & - & \\
\hline Ficat grading & -0.566 & $<0.001$ & -0.532 & $<0.001$ \\
\hline VAS scores & -0.555 & $<0.001$ & -0.513 & $<0.001$ \\
\hline HSSs & 0.404 & 0.001 & -0.368 & 0.002 \\
\hline WOMAC scores & -0.310 & 0.003 & 0.353 & 0.005 \\
\hline
\end{tabular}

Note: ${ }^{2}$ Adjusted by age and BMI.

Abbreviations: APN, adiponectin; HSS, Harris hip score; VAS, visual analog scale; WOMAC, Western Ontario and McMaster University Osteoarthritis Index; ONFH, osteonecrosis of the femoral head; BMl, body mass index.

APN levels were negatively associated with both the symptomatic and radiographic severities of nontraumatic ONFH. All the associations remained significant after adjusting for age and BMI. These results indicate that APN may play an important role in protecting ONFH and may serve as a novel and reliable biomarker for reflecting disease severity of ONFH. To the best of our knowledge, this is the first study to demonstrate the association of APN concentration in serum with the disease severity of nontraumatic ONFH. Our finding suggest that APN could possibly be used as a biomarker for clinical investigation.

In recent years, authors looking to embrace a consensus of opinion related to the pathogenesis of ONFH in their works have been devoting increasingly more attention to the importance of abnormal lipid metabolism. ${ }^{21}$ Research works over these years have supported the fact that ONFH is a problem of not merely local avascular status associated with bone necrosis but also including a systemic angle - in view of a significant contribution of hyperlipidemia. ${ }^{22}$ The 

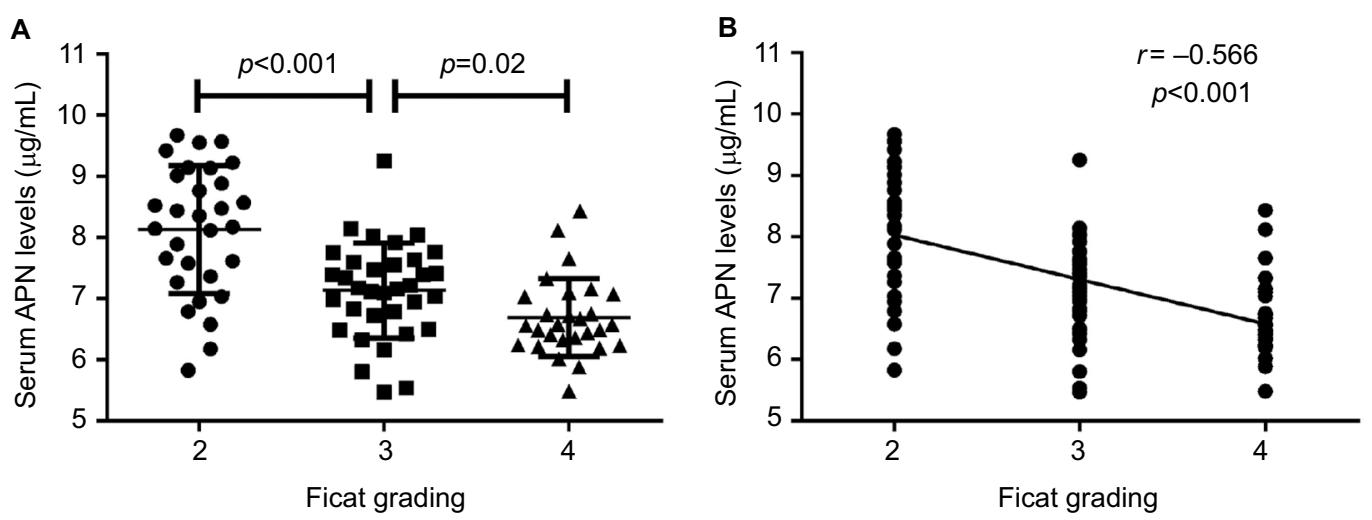

Figure 3 (A) Comparison of serum APN levels among different Ficat groups. (B) Correlation of serum APN levels with Ficat grading. Abbreviation: APN, adiponectin.
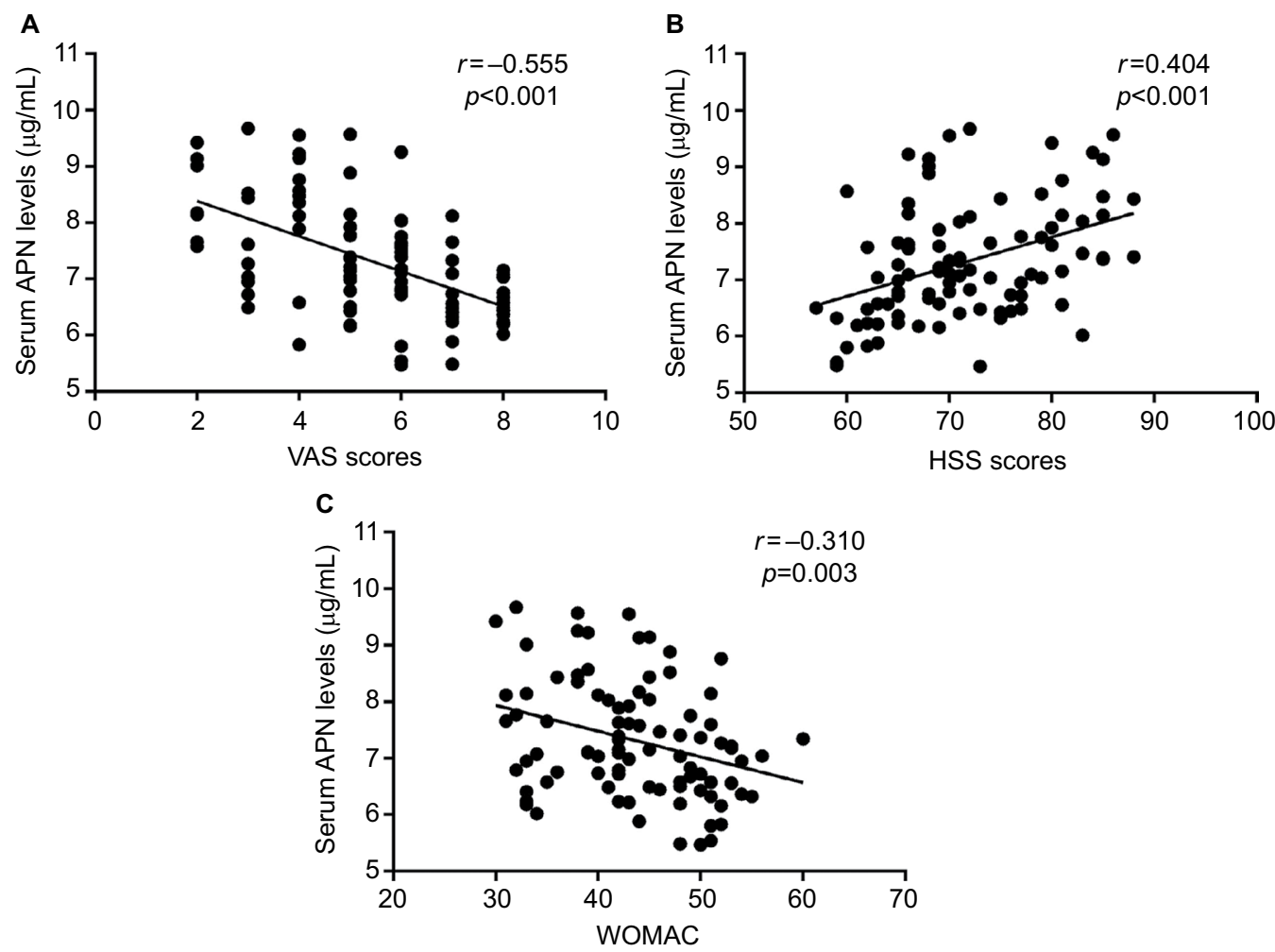

Figure 4 Correlation of serum APN levels with clinical severity determined by VAS scores (A), HSSs, (B) and WOMAC scores (C).

Abbreviations: APN, adiponectin; VAS, visual analog scale; HSS, Harris hip score; WOMAC, Western Ontario and McMaster University Osteoarthritis Index.

adipose tissue produces various bioactive factors, also known as adipokines. The imbalanced production of adipokines contributes to the abnormal lipid function. APN, the most widely studied adipose tissue protein, is a 244 amino acid, $30 \mathrm{kDa}$ peptide product of the apM1 gene. ${ }^{23} \mathrm{APN}$ has been intensely studied in many metabolic conditions such as insulin sensitivity, cardiovascular system diseases, and inflammatory and anti-inflammatory pathways. ${ }^{23,24}$

To date, APN has been investigated with its occurrence and potential involvement in ONFH only in a few studies. ${ }^{12}$
Low levels of APN and the positive correlation with inflammatory status and LDL/HDL ratio in ONFH patients suggest possible involvement of the metabolic pathways in the pathophysiology of the disease. ${ }^{12}$ However, studies that have investigated the role of APN in disease etiopathology of ONFH are not fully illustrated.

Recent studies have identified that APN functions as an important regulator of the fat-bone relationship and performs positive effects on lipid metabolism, thus playing a pivotal role in the bone metabolism. ${ }^{25,26}$ The two main receptors 
AdipoR1 and AdipoR2 of APN have been found to express on osteoblasts, osteoclasts, and bone marrow cells. ${ }^{7,11,27}$ APN has also been proven to induce osteoblast proliferation and differentiation through $\mathrm{p} 38 \mathrm{MAPK}$ signaling pathway, ${ }^{28}$ while inhibiting differentiation of osteoclasts and bone resorption activity, ${ }^{29}$ implicating that APN positively stimulates bone growth.

We first observed that APN levels in ONFH patients were decreased compared with healthy individuals. This is consistent with a previous published study. ${ }^{12} \mathrm{Next}$, we found APN levels decreased as Ficat grading increased, implicating that APN levels were associated with bone necrosis. We also found that circulating APN levels was negatively correlated with complaints of pain and functional disability determined by VAS scores, HSSs and WOMAC scores. Previous studies have identified that APN could locally regulate the transcription factor peroxisome proliferator-activated receptor- $\gamma(\operatorname{PPAR} \gamma),{ }^{30}$ which has been known to perform anti-hyperalgesic properties ${ }^{31}$ and inhibit the pain-related proinflammatory cytokines, such as TNF- $\alpha$ and IL- $1 \beta .{ }^{32,33}$ Other studies found that dysregulation of serum APN levels was correlated with chronic daily headache, ${ }^{34}$ variant angina, ${ }^{35}$ and coronary spastic angina, ${ }^{36}$ a form of chest pain.

There were some limitations that should be taken into account. First, this study was performed as a cross-sectional and single-centered study in China, although the statistics were powerful according to the enrolled numbers of subjects. Further multicenter studies should be carried out to identify the results. Second, we only measured APN levels in serum; other biochemical indices including lipid metabolism markers and cytokines were not investigated. Third, although circulating APN was proposed to be a useful biomarker to assess the progression of ONFH, sensitivity and specificity of serum APN level were not evaluated.

Collectively, we found lower levels of circulating APN in nontraumatic ONFH patients compared with healthy controls. In addition, APN levels were negatively related to radiological and symptomatic severity. Further studies investigating global hormone levels throughout the disease course of ONFH would provide a better insight about the potential clinical usefulness of APN.

\section{Acknowledgment}

Biao-Fang Wei is the guarantor of this study.

\section{Disclosure}

The authors report no conflicts of interest in this work.

\section{References}

1. Moya-Angeler J, Gianakos AL, Villa JC, Ni A, Lane JM. Current concepts on osteonecrosis of the femoral head. World J Orthop. 2015;6(8):590-601.

2. Aldridge JM, Urbaniak JR. Avascular necrosis of the femoral head: etiology, pathophysiology, classification, and current treatment guidelines. Am J Orthop. 2004;33:327-332.

3. Bradway JK, Morrey BF. The natural history of the silent hip in bilateral atraumatic osteonecrosis. J Arthroplasty. 1993;8(4):383-387.

4. Zalavras CG, Lieberman JR. Osteonecrosis of the femoral head: evaluation and treatment. J Am Acad Orthop Surg. 2014;22(7):455-464.

5. Magnussen RA, Guilak F, Vail TP. Articular cartilage degeneration in post-collapse osteonecrosis of the femoral head. Radiographic staging, macroscopic grading, and histologic changes. J Bone Joint Surg Am. 2005;87(6):1272-1277.

6. Qi X, Zeng Y. Biomarkers and pharmaceutical strategies in steroidinduced osteonecrosis of the femoral head: a literature review. $J$ Int Med Res. 2015;43(1):3-8

7. Berner HS, Lyngstadaas SP, Spahr A, et al. Adiponectin and its receptors are expressed in bone-forming cells. Bone. 2004;35(4):842-849.

8. Park PH, Sanz-Garcia C, Nagy LE. Adiponectin as an anti-fibrotic and anti-inflammatory adipokine in the liver. Curr Pathobiol Rep. 2015;3(4):243-252.

9. Lenchik L, Register TC, Hsu FC, et al. Adiponectin as a novel determinant of bone mineral density and visceral fat. Bone. 2003;33(4): 646-651.

10. Oshima K, Nampei A, Matsuda M, et al. Adiponectin increases bone mass by suppressing osteoclast and activating osteoblast. Biochem Biophys Res Commun. 2005;331(2):520-526.

11. Shinoda Y, Yamaguchi M, Ogata N, et al. Regulation of bone formation by adiponectin through autocrine/paracrine and endocrine pathways. J Cell Biochem. 2006;99(1):196-208.

12. Shuai B, Shen L, Yang YP, Xie J, Shou ZX, Wei B. Low plasma adiponectin as a potential biomarker for osteonecrosis of the femoral head. J Rheumatol. 2010;37(10):2151-2155.

13. Iannitti T, Graham A, Dolan S. Adiponectin-mediated analgesia and anti-inflammatory effects in rat. PLoS One. 2015;10(9):e0136819.

14. Jawad MU, Haleem AA, Scully SP. In brief: Ficat classification: avascular necrosis of the femoral head. Clin Orthop Relat Res. 2012;470: 2636-2639.

15. Harris WH. Traumatic arthritis of the hip after dislocation and acetabular fractures: treatment by mold arthroplasty. An end-result study using a new method of result evaluation. J Bone Joint Surg Am. 1969;51:737-755.

16. Shah SN, Kapoor CS, Jhaveri MR, Golwala PP, Patel S. Analysis of outcome of avascular necrosis of femoral head treated by core decompression and bone grafting. J Clin Orthop Trauma. 2015;6(3):160-166.

17. Bellamy N. WOMAC: a 20-year experiential review of a patient-centered self-reported health status questionnaire. J Rheumatol. 2002;29: 2473-2476.

18. Mao Q, Wang W, Xu T, et al. Combination treatment of biomechanical support and targeted intra-arterial infusion of peripheral blood stem cells mobilized by granulocyte-colony stimulating factor for the osteonecrosis of the femoral head: a randomized controlled clinical trial. J Bone Miner Res. 2015;30(4):647-656.

19. Tabatabaee RM, Saberi S, Parvizi J, Mortazavi SM, Farzan M. Combining concentrated autologous bone marrow stem cells injection with core decompression improves outcome for patients with early-stage osteonecrosis of the femoral head: a comparative study. J Arthroplasty. 2015;30(9 suppl):11-15.

20. Chow S, Shao J, Wang H. Sample Size Calculations in Clinical Research. 2nd ed. Boca Raton, FL: Chapman \& Hall/CRC Biostatistics Series; 2008:58.

21. Liu X, Li Q, Sheng J, et al. Unique plasma metabolomic signature of osteonecrosis of the femoral head. J Orthop Res. 2016;34(7): $1158-1167$. 
22. Moskal JT, Topping RE, Franklin LL. Hypercholesterolemia: an association with osteonecrosis of the femoral head. Am J Orthop (Belle Mead NJ). 1997;26(9):609-612.

23. Yang Y, Hu W, Jiang S, et al. The emerging role of adiponectin in cerebrovascular and neurodegenerative diseases. Biochim Biophys Acta. 2015;1852(9):1887-1894.

24. Ruan H, Dong LQ. Adiponectin signaling and function in insulin target tissues. J Mol Cell Biol. 2016;8(2):101-109.

25. Tamang HK, Timilsina U, Singh KP, et al. Assessment of adiponectin level in obese and lean Nepalese population and its possible correlation with lipid profile: a cross-sectional study. Indian J Endocrinol Metab. 2013;17(suppl 2):349-354.

26. Williams GA, Wang Y, Callon KE, et al. In vitro and in vivo effects of adiponectin on bone. Endocrinology. 2009;150(8):3603-3610.

27. Lee HW, Kim SY, Kim AY, Lee EJ, Choi JY, Kim JB. Adiponectin stimulates osteoblast differentiation through induction of COX2 in mesenchymal progenitor cells. Stem Cells. 2009;27(9):2254-2262.

28. Luo XH, Guo LJ, Yuan LQ, et al. Adiponectin stimulates human osteoblasts proliferation and differentiation via the MAPK signaling pathway. Exp Cell Res. 2005;309(1):99-109.

29. Tu Q, Zhang J, Dong LQ, et al. Adiponectin inhibits osteoclastogenesis and bone resorption via APPL1-mediated suppression of Akt1. J Biol Chem. 2011;286(14):12542-12553.
30. Fantuzzi G. Adiponectin in inflammatory and immune-mediated diseases. Cytokine. 2013;64:1-10.

31. Churi SB, Abdel-Aleem OS, Tumber KK, Scuderi-Porter H, Taylor $\mathrm{BK}$. Intrathecal rosiglitazone acts at peroxisome proliferator-activated receptor-gamma to rapidly inhibit neuropathic pain in rats. J Pain. 2008;9(7):639-649.

32. Beloeil H, Ji RR, Berde CB. Effects of bupivacaine and tetrodotoxin on carrageenan-induced hind paw inflammation in rats (Part 2): cytokines and p38 mitogen-activated protein kinases in dorsal root ganglia and spinal cord. Anesthesiology. 2006;105(1):139-145.

33. Huber JD, Campos CR, Mark KS, Davis TP. Alterations in bloodbrain barrier ICAM-1 expression and brain microglial activation after lambda-carrageenan-induced inflammatory pain. Am J Physiol Heart Circ Physiol. 2006;290(2):H732-H740.

34. Peterlin BL, Alexander G, Tabby D, Reichenberger E. Oligomerization state-dependent elevations of adiponectin in chronic daily headache. Neurology. 2008;70(20):1905-1911.

35. Hong SJ, Seo HS, Rha SW, et al. Decrease in plasma adiponectin concentrations in patients with variant angina pectoris. Circ J. 2006;70(4):414-418.

36. Maruyoshi H, Kojima S, Otsuka F, et al. Hypoadiponectinemia is associated with coronary artery spasm in men. Circ J. 2005;69(9):1154-1156.
Journal of Pain Research

\section{Publish your work in this journal}

The Journal of Pain Research is an international, peer reviewed, open access, online journal that welcomes laboratory and clinical findings in the fields of pain research and the prevention and management of pain. Original research, reviews, symposium reports, hypothesis formation and commentaries are all considered for publication.

\section{Dovepress}

The manuscript management system is completely online and includes a very quick and fair peer-review system, which is all easy to use. Visit http://www.dovepress.com/testimonials.php to read real quotes from published authors. 